\section{Prevenção de Morte Súbita em Portadores de Cardiomiopatia \\ Hipertrófica}

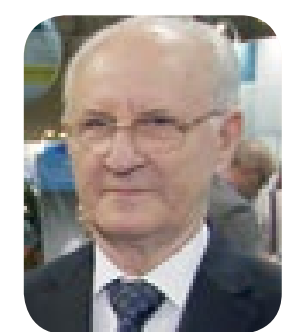

Gilson Feitosa

\section{INTRODUÇÃO}

Cardiomiopatia hipertrófica $(\mathrm{CMH})$ é a mais frequente das doenças estruturais relevantes de origem genética. Tem uma estimativa de ocorrência de $1 \mathrm{em}$ cada 500 indivíduos na população em geral.

Sua característica fenotípica mais comum expressa uma hipertrofia ventricular esquerda, predominantemente na porção proximal do septo interventricular causando um tensionamento do aparelho subvalva do folheto anterior da mitral durante a ejeção ventricular esquerda, gerando uma obstrução dinâmica da via de saída do ventrículo esquerdo, e eventual insuficiência mitral.

Sintomas e sinais correspondentes a este fenótipo incluem dispneia progressiva, dor precordial, síncope e achado de um sopro característico entre a reborda esternal esquerda e o foco mitral, mesossistólico e que aumenta com a manobra de Valsalva.

Sendo esta a apresentação mais reconhecida nem sempre se observa a presença do total espectro dessas manifestações ou achados.

Alguns pacientes são francamente assintomáticos e até mesmo esportistas de alta performance e que têm a condição reconhecida pelo achado de um sopro ou em ECG com SVE ou num ecocardiograma.

Outros fenótipos incluem hipertrofia generalizada apical, entre outras.

Em todas as formas paira uma preocupação de morte súbita, que a rigor parecia de início ter maior prevalência do que a que hoje se registra, sendo atualmente estimada entre 1 e $2 \%$ ao ano.?

Uma avaliação da razão da mudança foi a seleção de pacientes, já que aos centros que colimavam as informações que originavam as publicações eram referidos os casos mais graves.

A ocorrência de morte súbita é observada em praticamente todas as faixas etárias e sendo mais prevalente entre os mais jovens. (Fig. 1) taquicardia ventricular e fibrilação ventricular. microrreentradas como sua origem. (Fig.
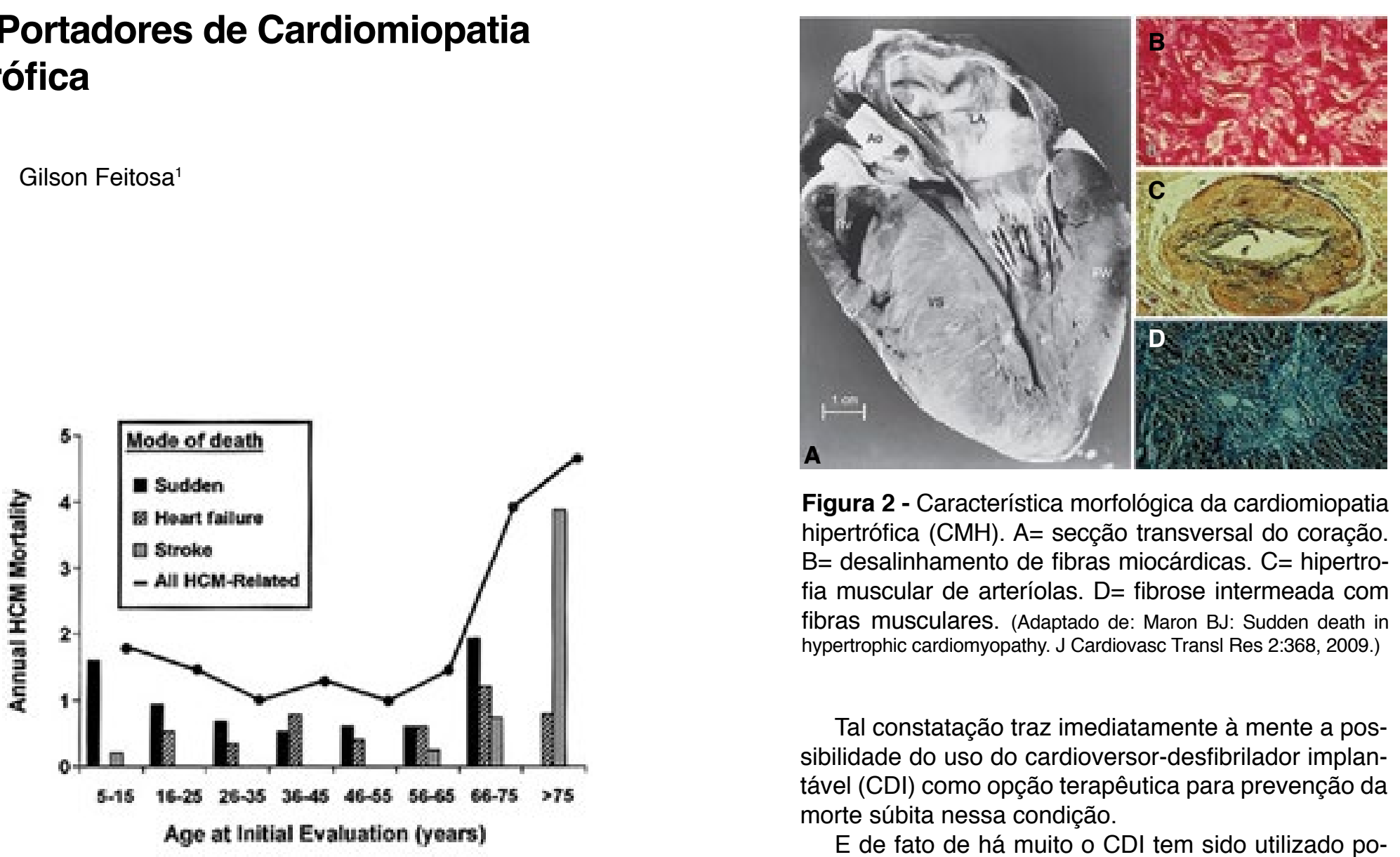

Figura 1 - Mortalidade e causa de óbito em portadores de CMH. (Adaptado de Maron B et al. Epidemiology of Hypertro-
phic Cardiomyopathy-Related Death Circulation. 2000;102:858-864)

A característica da morte súbita em casos de $\mathrm{CMH}$ geralmente abrupla e a farta documentaçáo em casos monitorizados durante sua ocorrência, confirmam suspeita de sua relação com arritmias ventriculares-

Bases histopatológicas que fundamentam esta tendência são claramente demonstradas em estudos de necropsia que revelam desalinhamento das fibras hipertrofiadas, em meio a quantidades variáveis de fibrose, ensejando a formação de sítios que propiciam
Figura 2 - Característica morfológica da cardiomiopatia hipertrófica $(\mathrm{CMH})$. $A=$ secção transversal do coração. $B=$ desalinhamento de fibras miocárdicas. $C=$ hipertrofia muscular de arteríolas. $D=$ fibrose intermeada com fibras musculares. (Adaptado de: Maron BJ: Sudden death in
hypertrophic cardiomyopathy. J Cardiovasc Transl Res 2:368, 2009.)

Tal constatação traz imediatamente à mente a possibilidade do uso do cardioversor-desfibrilador implantável (CDI) como opção terapêutica para prevenção da morte súbita nessa condição.

E de fato de há muito $O \mathrm{CDI}$ tem sido utilizado podendo-se dele confirmar sua alta eficácia em prevenir morte súbita de portadores de $\mathrm{CMH}$.

Em um acompanhamento de 5 anos de uma série morte súbita foi constatado, sendo que no interrogatório da memória desses aparelhos houve um considerável número de choques apropriados, isto é, choques fibrilação ventricular, numa taxa de $7,9 \%$ ao ano em casos de prevenção secundaria e de $4,0 \%$ ao ano em casos de prevenção primária, mostrando o acerto do seu uso neste grupo de pacientes avaliados. ${ }^{3}$

Porém, o seu uso não transcorreu sem o aparecimento de efellos indesejaveis, tais como choques inapropriados em $33,7 \%$ dos casos, por motivos outros que não as artitmias venticulares, a exemplo de tremores musculares, episódios de fibrilação atrial ou taquicardia atrial, entre outros, e que promovem considerável desconforto ao paciente, afetando assim sua qualidade de vida. ${ }^{2}$

Por outro lado, registraram-se falência de eléctrodos ou infecção de partes do sistema em 12,5\% e de 104 pacientes com CMH e CDI, nenhum caso de que reverteram episódios de taquicardia ventricular ou
$4,8 \%$, respectivamente, com as implicações mórbidas consequentes.

O que nos remete à consideração de se procurar dentificar os pacientes que mais se beneficiarão deste recurso.

A ESCOLHA DO CANDIDATO AO USO DO CDI

Quando se trata de prevenção secundária de morte súbita em portadores de CMH, a escolha é obvia, tendo em vista a alta incidência deste evento nesta condição. São candidatos à prevenção secundária os pacientes que foram ressuscitados de um episodio de parada cardiaca ou aqueles que demonstrem em um Holter a presença de taquicardia ventricular por mais de 30 batimentos e com frequência cardíaca maior que $120 \mathrm{bpm}$. Quando um portador de CMH não apresenta as ocorrências acima e, ainda assim, recebe um CDI para prevenção primária.

Há muito se reconhece que certos achados, quando presentes, expõem o portador de $\mathrm{CMH}$ a uma chance maior de morte súbita, sendo que via de regra os mais considerados são: síncope inexplicada, história familiar de morte súbita em jovens, espessura septa maior que $30 \mathrm{~mm}$, resposta anormal da pressão arterial ao exercício e presença de taquicardia ventricula não sustentada (TVNS)

A presença de vários desses fatores num pacient configura-lhe um risco elevado para morte súbita.

Ocorre, porém, que, entre os que foram ressuscita-

dos, ou quando já em uso de CDI receberam choques apropriados, é relativamente infrequente a presença simultânea destes fatores.

E ainda assim tiveram morte súbita havendo a necessidade de melhor identificação da importância destes fatores.

Individualmente em ordem decrescente de razão de chances (HR; $(95 \%$ IC)de predição de morte súb ta temos: espessura septal $>30 \mathrm{~mm}=3,1(1,81-4,40)$ TVNS 2,89 $(2,21-3,58)$; síncope inexplicada 2,68 $(0,97-4,38) ; 1,30(0,64-1,96)$ e história familiar de morte súbita $1,27(1,16-1,38)$.

Ao mesmo tempo há uma certa linearidade entre espessura septal e morte súbita, não se tratando, portanto, de uma opção dicotomizada de mais ou menos que $30 \mathrm{~mm}$

Alem disso, a presença individual de cada um dos fatores, em casos de morte subita, variou de 9 a $30 \%$.

Tais constataçoes levaram à construção de escores de risco, tomando em conta a presença e força de representação de variáveis lógicas levantadas, sendo que o mais recente e utilizado tem sido o Escore de Risco 
para Morte Súbita em CMH - (HCM Risk-SCD) da Sociedade Europeia de Cardiologia, que deduziu um escore prognóstico para morte súbita por meio da análise
retrospectiva de 6 coortes de pacientes em 6 centros retrospectiva de 6 coortes de pacientes em 6 centros europeus, compreendendo um total de 3.066 pacientes numa mediana de acompanhamento de 5,7 anos, com

ocumentação de morte súbita em 198 casos.

Os fatores identificados foram idade (anos), espessura máxima do septo interventricular $(\mathrm{mm})$, diâmetro atrial esquerdo $(\mathrm{mm})$, gradiente maximo na via de saída do ventrículo esquerdo $(\mathrm{mmHg})$, historia familiar de morte súbita, taquicardia ventricular não sustentada Holter de 24h e sincope sem outra causa.

Tais fatores foram obtidos numa análise inicial de regressão univariada com exigência maior de significância de 15\% (habitualmente, 10\%) para compens questões porventura ligadas ao número da amostra. Daí a disponibilização de um calculador on-line, que permite a predição anual de risco de morte súbita (wWw.HCMRs.org/. hth://Www.doc2do.com/hcm/ webHCM.html) e que recebe o endorso da Sociedade uropeia de Cardiologia.

A estimativa de risco de morte súbita por esse escore em 5 anos orienta a conduta quanto ao implante de um CDI para prevenção primária, de acordo com a tabela abaixo:

ESTIMATIVA DE RISCO PARA MORTE SÚBITA EM 5 ANOS - Baixo risco $(<4 \%)$ - Risco intermediário - Alto rico $(>6 \%)$

$\cdot$ CDI não indicado $\begin{gathered}\cdot \begin{array}{c}\text { CDI pode ser } \\ \text { considerado }\end{array} \\ \cdot \mathrm{CDI} \text { indicado }\end{gathered}$

Este escore teve uma validação externa em população europeia (REF) onde demonstrou ter numa coorte de 706 pacientes, num acompanhamento de mais de 7 anos, uma estatistica $C$ de predição de 0,69, superior à da Diretriz ACC/ESC de 2003, de 0,55, e a da ACCF/AHA de 2011, de 0,60. ${ }^{6}$

PERSPECTIVAS DO USO DARESSONÂNCIAMAGNÉTICA CARDÍACA NA ESTRATIFICAÇÃO DE RISCO PARA MORTE SÚBITA NO PORTADOR DE CMH

É claramente reconhecido por todos que escores de estimativa de risco não apresentam uma precisão perfeita, sendo, no entanto, frequentemente, o melhor recurso de que se dispõe para o enquadramento de certos casos.

No caso do escore europeu de predição de risco de morte súbita, encontram-se questionamentos quanto à sua sensibilidade na estimativa de risco, principalmente oriundos das observações realizadas em centros especializados para tratamento dessa doença no tados Unidos.

Com o advento da imagem obtida por ressonância magnética cardíaca e seu reconhecido papel em revelar a presença de fibrose e determinar prognóstico em cardiopatia isquêmica, trouxe a atenção para o seu mprego em HCM.

Trabalhos pioneiros de Rochite e seu grupo no Brasil ${ }^{7}$ documentavam seu possível papel e, mais recentemente, um trabalho muito expressivo nesta área, realizado por Chan e cols.", num acompanhamento de 1.293 pacientes, dos quais $3 \%$ vieram a ter morte súbita num acompanhamento de 3 anos, notou-se um incremento de $46 \%$ de aumento de morte súbita por cada aumento de $10 \%$ no percentual de fibrose, em relação à massa de ventrículo esquerdo.

Destacou-se a observação de que o risco parecia bastante expressivo quando o percentual de fibrose excedia os $15 \%$ da massa ventricular.

No momento, essa informação está sendo consderada por alguns como útil para aumentar a sensibidade de indicação do CDI para quem apresente um score abaixo de 6 no escore europeu.

Outros aventam a hipótese de seu emprego para talvez diminuir a indicação de CDI em quem tenha escore elevado, e percentual baixo de fibrose, em bora essa linha de pensamento encontre maiores resistências.

De qualquer forma, se prevê em próximas diretrizes sobre o assunto de CMH um papel bem mais destacado da ressonância magnética na predição prognóstica de morte súbita nesta condição.9,

\section{CONCLUSÃO}

Embora infrequente, a morte súbita representa uma ameaça a alguns portadores de $\mathrm{CMH}$.

O conveniente emprego do $C D I$ representa sua mehor forma de tratamento preventivo dessa condição. A escolha de quem mais se beneficiará de sua utlização em prevenção primaria depende da adequada identificação das características do paciente por meio do reconhecimento de achados de risco para essa ocorrencia e, principalmente, com o uso de escores de predição, sendo aquele mais em voga o da Sociedade Europeia de Cardiologia.

A quantidade de fibrose demonstrada na ressonância magnetica cardíaca pode vir a se constituir num auxílio à identificação do paciente de maior risco.
REFERÊNCIAS

1. Loar RW, Bos JM, Will ML, Ommen SR, Ackerman MJ. Genotype-phenotype Correlations of Hypertrophic Cardiomyopathy When Diagnosed in Children Adolescents, and Young Adults. Congenit Heart Dis 2015 November; 10(6):529-36.

2. Maron BJ. Historical Perspectives on Sudden Deaths in Young Athletes With Evolution over 35 Years. Am J Cardiol 2015 November 1;116(9):1461-8.

3. Syska P, Przybylski A, Chojnowska $L$ et al. Implantable cardioverter-defibrillator in patients with hypertrophic cardiomyopathy: efficacy and complications of the therapy in long-term follow-up. J Cardiovasc Electrophysiol 2010 August 1;21(8):883-9.

4. Magnusson P, Morner S, Gadler F, Karlsson J. Health-related quality of life in hypertrophic cardiomyopathy patients with implantable defibrillators. Health ual Life Outcomes 2016 April 14;14:62.

5. Elliott PM, Anastasakis A, Borger MA et al. 2014 ESC Guidelines on diagnosis and management of hypertrophic cardiomyopathy: the Task Force for the Diagnosis and Management of Hypertrophic Cardiomyopathy of the European Society of Cardiology (ESC). Eur Heart J 2014 October 14;35(39):2733-79. 6. Vriesendorp PA, Schinkel AF, Liebregts $\mathrm{M}$ et al. Validation of the 2014 European Society of Cardiology guidelines risk prediction model for the primary prevention of sudden cardiac dealh in hypertrophic cardiomyopathy. Circ Arrhythm Electrophysiol $2015 \mathrm{Au}$ gust;:8(4):829-35

7. Shiozaki AA, Senra T, Arteaga E et al. Myocardia fibrosis detected by cardiac CT predicts ventricular fibrillation/ventricular tachycardia events in patients with hypertrophic cardiomyopathy. J Cardiovasc Comput Tomogr 2013 May;7(3):173-81

8. Chan RH, Maron BJ, Olivotto I et al. Prognostic value of quantitative contrast-enhanced cardiovascular magnetic resonance for the evaluation of sudden death risk in patients with hypertrophic cardiomyopathy. Circulation 2014 August 5;130(6):484-95.

9. Rowin EJ, Maron MS. The Role of Cardiac MR in the Diagnosis and Risk Stratification of Hypertrophic Cardiomyopathy. Arrhythm Electrophysiol Rev 2016;5(3):197-202.

10. Oliveira DC, Assunção FB, Santos AA, Naci MS. Cardiac Magnetic Resonance and Computed Tomography in Hypertrophic Cardiomyopathy: an Update. Arq Bras Cardiol 2016 August:107(2):163-72.
1- Serviço de Cardiologia Clínica do Hospital Santa

Endereço para correspondência:

gilson-feitosa@uol.com.br 